\section{Kidney \\ Blood Pressure Research}

Kidney Blood Press Res 2012;36:290-300

\begin{tabular}{l|l}
\hline DOI: $10.1159 / 000343418$ & (c) 2012 S. Karger AG, Basel
\end{tabular}

Published online: December 12, 2012

www.karger.com/kbr

$1420-4096 / 12 / 0361-0290 \$ 38.00 / 0$

\title{
Determining the Optimal Cut-Off Value of the Urinary Albumin-To-Creatinine Ratio to Detect Atherosclerotic Vascular Diseases
}

\author{
Young-Hoon Lee ${ }^{a, b}$ Sun-Seog Kweon ${ }^{c, d} \quad J i n-S u$ Choic Jung-Ae Rhee \\ Hae-Sung Name Seul-Ki Jeong ${ }^{\mathrm{f}}$ Kyeong-Soo Park ${ }^{g}$ Hye-Yeon Kim ${ }^{9}$ \\ So-Yeon Ryu ${ }^{\text {h }}$ Seong-Woo Choi ${ }^{\text {h }}$ Bok-Hee Kim ${ }^{i}$ Min-Ho Shin ${ }^{c}$
}

aDepartment of Preventive Medicine \& Institute of Wonkwang Medical Science, Wonkwang University College of Medicine, Iksan, Jeonbuk, South Korea, 'begional Cardiocerebrovascular Center, Wonkwang University Hospital, Iksan, Jeonbuk, South Korea, 'Department of Preventive Medicine, Chonnam National University Medical School, Gwangju, South Korea, dJeonnam Regional Cancer Center, Chonnam National University Hwasun Hospital, Hwasun, South Korea, eDepartment of Preventive Medicine, Chungnam National University College of Medicine, Daejeon, South Korea, fDepartment of Neurology, Chonbuk National University-Chonbuk National University Hospital, Jeonju, Jeonbuk, South Korea, ${ }^{9}$ Department of Preventive Medicine, Seonam University College of Medicine, Namwon, Jeonbuk, South Korea, hDepartment of Preventive Medicine, Chosun University Medical School, Gwangju, South Korea, 'Department of Food \& Nutrition, Chosun University College of Natural Sciences, Gwangju, South Korea

\section{Key Words}

Albuminuria • Atherosclerosis • Carotid artery • Arterial stiffness

\begin{abstract}
Background: We examined whether low-grade albuminuria, below the conventional cut-off value for microalbuminuria, was associated with atherosclerotic vascular diseases in 8897 community-dwelling Koreans aged $\geq 50$ years. Methods: The urinary albumin-to-creatinine ratio (UACR) was calculated using random spot urine. Common carotid artery (CCA) intimamedia thickness (IMT) and CCA internal diameter were measured using high-resolution B-mode ultrasonography, and carotid plaque was evaluated. Brachial-ankle pulse wave velocity (BaPWV) and the ankle-brachial index (ABI) were examined, and peripheral arterial disease was defined as ABI <0.9. Results: Youden's indices, predicting abnormal atherosclerotic conditions, were greatest at a UACR cut-off value of $\sim 15 \mathrm{mg} / \mathrm{g}$, below the threshold conventionally used to define microalbuminuria. Compared with low normoalbuminuria (UACR $<15.0 \mathrm{mg} / \mathrm{g}$ ), CCA IMT, CCA diameter, and BaPWV were significantly greater in individuals with high normoalbuminuria (UACR $15.0-29.9 \mathrm{mg} / \mathrm{g}$ ), who also had a significantly higher risk of carotid plaque than did
\end{abstract}




\section{Kidney Blood Pressure Research}

those with low normoalbuminuria. Conclusions: Subclinical atherosclerotic vascular diseases developed at lower UACRs, below the conventional classification of microalbuminuria. Further longitudinal studies are needed to investigate the relationship between microalbuminuria and the development of subclinical atherosclerosis.

Copyright (C) 2012 S. Karger AG, Basel

\section{Introduction}

Microalbuminuria is defined as a urinary albumin-to-creatinine ratio (UACR) of 30 to $<300 \mathrm{mg} / \mathrm{g}$ in the first midstream urine specimen in the morning [1]. Recent epidemiological studies have demonstrated that microalbuminuria is an independent risk factor for cardiovascular morbidity and mortality and all-cause mortality in the general population without hypertension or diabetes [2,3]. Even levels of albuminuria below the conventional cut-off value for microalbuminuria have been found to be significantly associated with cardiovascular morbidity and mortality [4]. Recently, many investigators have suggested revision of the microalbuminuria cut-off value to increase the sensitivity of detecting subjects at high risk [5].

Epidemiological studies have examined significant associations of microalbuminuria with carotid atherosclerosis in high-risk populations with hypertension or diabetes [68], and also in the general population [9]. Moreover, lower UACRs compared with the conventional cut-off value are significantly associated with carotid atherosclerosis $[9,10]$. Microalbuminuria is significantly associated with arterial stiffness, not only in high-risk individuals with hypertension or diabetes $[11,12]$, but also in the general population $[13,14]$. Most previous studies have examined the association between albuminuria and subclinical atherosclerosis in high-risk individuals, whereas only a few studies have investigated such an association in the general population. In addition, few studies have investigated the association of albuminuria with various atherosclerotic vascular diseases simultaneously in a large community-dwelling population. Our previous study found that the estimated glomerular filtration rate (eGFR) was associated with atherosclerosis, which was evaluated with carotid plaque, peripheral arterial disease (PAD), and brachial-ankle pulse wave velocity (baPWV) in Koreans age 50 years and older [15].

Therefore, in this study, we investigated the association of albuminuria measured by UACR with overall atherosclerotic vascular parameters such as carotid intima-media thickness (IMT), plaque, diameter, pulse wave velocity, and ankle-brachial index (ABI). Moreover, we examined whether low-grade albuminuria, below the conventional cut-off value for microalbuminuria, was associated with atherosclerotic vascular diseases in a large general population.

\section{Materials and Methods}

\section{Study Population}

The individuals included were participants in the baseline survey of the Dong-gu Study conducted between 2007 and 2010. The Dong-gu Study is an ongoing population-based prospective study evaluating the prevalence, incidence, and risk factors for chronic disease in the urban elderly population. We used the national resident register to identify potential participants and 34,040 eligible individuals aged $\geq 50$ years who resided in the Dong-gu district of Gwangju, Korea, were invited to participate by mail and telephone. Of these, a total of 9260 were enrolled (response rate, 27.2\%). In the present analyses, 363 were excluded because of missing information for UACR or atherosclerosis. The final sample consisted of 8897 individuals (3564 men and 5333 women). The study was performed in accordance with the Second Helsinki Declaration, and informed consent for the procedure was obtained from each participant. The study protocol was approved by the Institutional Review Board of Chonnam National University Hospital. 


\section{Kidney Blood Pressure Research}

Lee/Kweon/Choi et al.: Albuminuria and Atherosclerosis

\section{Urinary albumin excretion}

Spot midstream urine specimens were collected from each individual in the morning. The urinary albumin concentration was determined by turbidimetric immunoassay and the urinary creatinine concentration was measured with a modified Jaffe method (model 7600 chemical analyzer; Hitachi, Tokyo, Japan). According to the conventional cut-off value, participants were categorized into three groups according to UACR: normoalbuminuria ( $<30.0 \mathrm{mg} / \mathrm{g}$ ), microalbuminuria (30.0-299.9 mg/g), and macroalbuminuria ( $\geq 300.0 \mathrm{mg} / \mathrm{g})$.

\section{Atherosclerotic vascular parameters}

IMT was measured as the distance from the media-adventitia interface to the intima-lumen interface on the far wall in a longitudinal view because of its high degree of clarity and reliability. The common carotid artery (CCA) IMT was determined as the average of the maximum IMT of the left and right CCAs. The reader also assessed the presence of carotid plaques, which were defined as focal structures that encroached into the lumen by at least $100 \%$ of the surrounding IMT value. The presence of carotid plaques was recorded if at least one lesion was detected in any segment in both carotid arteries. The CCA diameter was determined as the mean of the internal diameter of the left and right CCAs at a point $10 \mathrm{~mm}$ proximal to the carotid bulb origin. Abnormal CCA IMT was defined as $\geq 1.0 \mathrm{~mm}$ and abnormal CCA diameter was defined as the 5 th quintile of CCA diameter because of the absence of standard values.

BaPWV and ABI were determined using an automated, noninvasive, waveform analysis device (VP1000; Colin, Komaki, Japan). Abnormal BaPWV was defined as the 5th quintile of BaPWV. The ABI was automatically calculated by dividing the posterior tibial systolic blood pressure by the brachial systolic pressure, measured by the oscillometric method with cuffs adapted to the extremities. An ABI was obtained for each leg separately, and the lower of the right and left ABIs was determined and used in subsequent analyses. PAD, or abnormal ABI, was defined as ABI $<0.9$ in either leg [16].

\section{Covariates}

Smoking status was classified into never, former, and current smoker. Alcohol intake was divided into never, former, and current drinker. Blood samples were drawn from an antecubital vein in the morning after a 12-h overnight fast. All samples were analyzed using an automatic analyzer (model 7600 chemical analyzer; Hitachi, Tokyo, Japan). Blood pressure was measured on the left upper arm using a mercury sphygmomanometer, with the person in a seated position after 5 min of rest. Three consecutive measurements were performed at 1-min intervals, and the average of three readings was recorded. Hypertension was defined as systolic blood pressure $\geq 140 \mathrm{mmHg}$, or diastolic blood pressure $\geq 90 \mathrm{mmHg}$, and/or use of antihypertensive medication. Diabetes was defined as fasting glucose $\geq 126 \mathrm{mg} / \mathrm{dL}$ and/or use of hypoglycemic medication or insulin.

\section{Statistical Analysis}

General characteristics of the study population, based on the conventional three UACR categories, were analyzed using analysis of variance for continuous variables and the $\chi^{2}$ test for categorical variables, and were expressed as mean \pm standard deviation or $n(\%)$. The conventional normoalbuminuria group was segmented into six categories: UACR <4.9, 5.0-9.9, 10.0-14.9, 15.0-19.9, 20.0-24.9, and 25.0-29.9 mg/g. All six segmented categories were compared with microalbuminuria to determine the association between UACR and subclinical atherosclerosis. After adjusting for age, sex, body mass index (BMI), smoking status, alcohol consumption, hypertension, diabetes, total/HDL cholesterol ratio, use of lipid-lowering medication and uric acid, mean (95\% CI) CCA IMT, CCA diameter, and BaPWV, and prevalence of carotid plaque and PAD were calculated. Linear trends in the association between segmented UACR categories and subclinical atherosclerotic conditions were also evaluated.

The ROC curve was used to evaluate the ability of the UACR to classify atherosclerotic vascular disease, and the optimal UACR cut-off value was determined using Youden's index. Adding the new UACR cut-off value of $15.0 \mathrm{mg} / \mathrm{g}$, associations between the revised classification based on UACR (low normoalbuminuria, high normoalbuminuria, microalbuminuria, macroalbuminuria) and atherosclerotic conditions were compared using a general linear model and multiple logistic regression model. All statistical analyses were performed using SPSS version 18.0 (SPSS Inc.). A value of $P<0.05$ was considered statistically significant. 


\section{Kidney \\ Blood Pressure Research}

Table 1: Characteristics of the study population according to the conventional classification based on the urinary albumin-to-creatinine ratio (UACR)

\begin{tabular}{lccc}
\hline & $\begin{array}{c}\text { Normoalbuminuria } \\
\text { [UACR }<30.0 \mathrm{mg} / \mathrm{g}] \\
(n=7099)\end{array}$ & $\begin{array}{c}\text { Microalbuminuria } \\
\text { [UACR 30.0-299.9 mg/g] } \\
(n=1618)\end{array}$ & $\begin{array}{c}\text { Macroalbuminuria } \\
{[\text { UACR } \geq 300.0 \mathrm{mg} / \mathrm{g}]} \\
(n=180)\end{array}$ \\
\hline Age, years & $64.5 \pm 7.9$ & $67.7 \pm 8.7^{*}$ & $67.8 \pm 8.1^{*}$ \\
Men, $n$ (\%) & $2783(39.2)$ & $696(43.0)^{*}$ & $85(47.2)^{*}$ \\
Body mass index, kg/m ${ }^{2}$ & $24.3 \pm 2.9$ & $24.7 \pm 3.0^{*}$ & $24.6 \pm 3.1$ \\
Waist circumference, cm & $87.8 \pm 8.6$ & $89.0 \pm 8.4^{*}$ & $89.7 \pm 9.1^{*}$ \\
Systolic blood pressure, mmHg & $121.5 \pm 15.9$ & $130.4 \pm 18.1^{*}$ & $135.4 \pm 19.1^{*}$ \\
Diastolic blood pressure, mmHg & $73.8 \pm 9.8$ & $76.7 \pm 11.3^{*}$ & $75.6 \pm 11.5$ \\
Fasting blood glucose, mg/dL & $106.7 \pm 20.3$ & $119.5 \pm 34.1^{*}$ & $136.9 \pm 47.3^{*}$ \\
Total cholesterol, mg/dL & $200.6 \pm 38.8$ & $203.5 \pm 43.8^{*}$ & $208.7 \pm 47.0^{*}$ \\
HDL cholesterol, mg/dL & $51.8 \pm 11.8$ & $50.8 \pm 12.3^{*}$ & $49.9 \pm 12.9$ \\
Triglycerides, mg/dL & $115[82-166]$ & $130[91-193]^{*}$ & $141[101-210]^{*}$ \\
Urea nitrogen, mg/dL & $16.5 \pm 4.5$ & $17.4 \pm 5.5^{*}$ & $21.4 \pm 11.3^{*}$ \\
Creatinine, mg/dL & $0.99 \pm 0.20$ & $1.06 \pm 0.35^{*}$ & $1.45 \pm 1.17^{*}$ \\
Uric acid, mg/dL & $4.97 \pm 1.35$ & $5.07 \pm 1.50^{*}$ & $5.88 \pm 1.95^{*}$ \\
Hypertension, $n$ (\%) & $2853(40.2)$ & $1000(61.8)^{*}$ & $127(70.6)^{*}$ \\
Diabetes, $n$ (\%) & $1040(14.6)$ & $542(33.5)^{*}$ & $109(60.6)^{*}$ \\
Lipid-lowering medication, $n(\%)$ & $592(8.3)$ & $141(8.7)$ & $24(13.3)^{*}$ \\
Smoking status, $n$ (\%) & & & \\
$\quad$ Never & $4877(68.7)$ & $1076(66.5)^{*}$ & $108(60.0)^{*}$ \\
Former & $1443(20.3)$ & $380(23.5)$ & $46(25.6)$ \\
Current & $779(11.0)$ & $162(10.0)$ & $26(14.4)$ \\
Alcohol drinking, $n$ (\%) & & & $81(45.0)^{*}$ \\
$\quad$ Never & $3214(45.3)$ & $712(44.0)$ & $28(15.6)$ \\
Former & $602(8.5)$ & $159(9.8)$ & $71(39.4)$ \\
Current & $3283(46.2)$ & $747(46.2)$ &
\end{tabular}

Values are mean \pm standard deviation or median [interquartile range] or $n(\%)$.

HDL, high-density lipoprotein.

" $P<0.05$ compared with normoalbuminuria.

Fig. 1. Urinary albuminto-creatinine ratio (UACR) accord-ing to systolic and diastolic blood pressure.

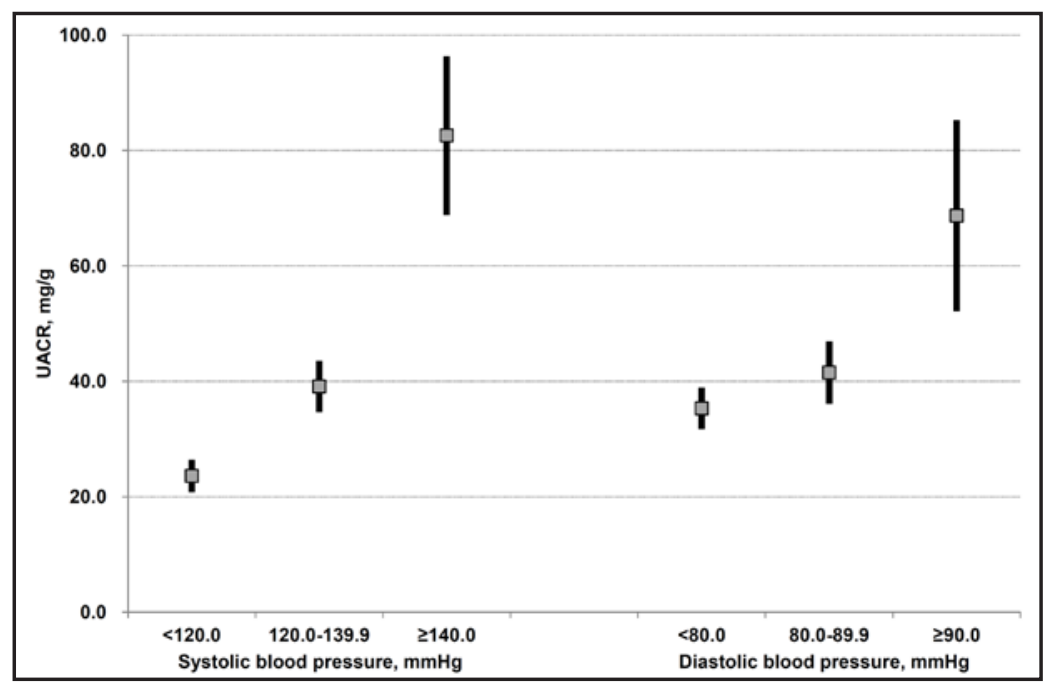

\section{Results}

Characteristics of the study population

Baseline characteristics according to the conventional classification based on UACR are summarized in Table 1 and 2. Of the 8897 people analyzed, 3564 (40.1\%) were men and $5333(59.9 \%)$ were women. Compared with those with normoalbuminuria, individuals with 


\section{Kidney Blood Pressure Research}

Table 2: Diagnostic performance of the urinary albumin-to-creatinine ratio (UACR) in predicting abnormal atherosclerotic vascular diseases using receiver operating characteristic curves

\begin{tabular}{lccccc}
\hline & $\begin{array}{c}\text { Abnormal } \\
\text { CCA IMT }\end{array}$ & $\begin{array}{c}\text { Abnormal } \\
\text { CCA diameter }\end{array}$ & $\begin{array}{c}\text { Abnormal } \\
\text { BaPWV }\end{array}$ & $\begin{array}{c}\text { Carotid } \\
\text { plaques }\end{array}$ & PAD \\
\hline AUC $(95 \% \mathrm{CI})$ & 0.578 & 0.625 & 0.675 & 0.584 & 0.618 \\
& $(0.560-0.596)$ & $(0.610-0.639)$ & $(0.661-0.689)$ & $(0.572-0.597)$ & $(0.582-0.655)$ \\
\hline Youden's index & & & & 0.040 & 0.051 \\
\hline $5 \mathrm{mg} / \mathrm{g}$ & 0.043 & 0.050 & 0.084 & 0.116 & 0.125 \\
$10 \mathrm{mg} / \mathrm{g}$ & 0.091 & 0.155 & 0.221 & 0.132 & 0.193 \\
$15 \mathrm{mg} / \mathrm{g}$ & 0.120 & 0.193 & 0.253 & 0.120 & 0.169 \\
$20 \mathrm{mg} / \mathrm{g}$ & 0.110 & 0.178 & 0.250 & 0.100 & 0.164 \\
$25 \mathrm{mg} / \mathrm{g}$ & 0.099 & 0.176 & 0.232 & 0.093 & 0.167 \\
$30 \mathrm{mg} / \mathrm{g}{ }^{\dagger}$ & 0.089 & 0.152 & 0.205 & 0.133 & 0.206 \\
Optimal (UACR) ${ }^{*}$ & 0.125 & 0.200 & 0.255 & $(14.7 \mathrm{mg} / \mathrm{g})$ & $(18.4 \mathrm{mg} / \mathrm{g})$ \\
\hline
\end{tabular}

CCA, common carotid artery; IMT, intima-media thickness; BaPWV, brachial-ankle pulse wave velocity; PAD, peripheral arterial disease; AUC, area under the curve; $\mathrm{CI}$, confidence interval.

Abnormal CCA IMT, maximal CCA IMT $\geq 1.0 \mathrm{~mm}$; abnormal CCA diameter, maximal CCA diameter in 5th quintile; abnormal BaPWV, maximal BaPWV in 5th quintile; PAD, minimal ankle-brachial index $<0.9$.

"Youden's index = sensitivity + specificity -1 .

†Conventional cut-off value for microalbuminuria.

¥0ptimal cut-off value that maximized Youden's index.

microalbuminuria were significantly older and had higher BMI, waist circumference, systolic and diastolic blood pressure, fasting blood glucose, triglycerides, urea nitrogen, creatinine, and uric acid, and lower HDL cholesterol. The prevalence of men, hypertension, and diabetes mellitus was significantly higher in subjects with microalbuminuria than in those with normoalbuminuria (Table 1). The UACR levels became significantly greater as the systolic and diastolic blood pressures increased (Figure 1).

Atherosclerotic conditions according to the segmented UACR classification

The conventional normoalbuminuria group was divided into six categories: UACR $<5.0,5.0-9.9,10.0-14.9,15.0-19.9,20.0-24.9$, and 25.0-29.9 mg/g. A significant difference for adjusted CCA IMT was observed only in those with UACR $<5.0 \mathrm{mg} / \mathrm{g}$, compared with microalbuminuria. Adjusted internal diameter of the CCA differed significantly in people in all UACR categories, except 25.0-29.9 mg/g, compared with microalbuminuria. Significant differences of adjusted BaPWV were observed in those with UACR <5.0, 5.0-9.9, 10.0-14.9, and $15.0-19.9 \mathrm{mg} / \mathrm{g}$, compared with microalbuminuria (Figure 2). The adjusted prevalence of carotid plaques in people with UACR $<5.0$ and $5.0-9.9 \mathrm{mg} / \mathrm{g}$ was significantly lower than in those with microalbuminuria. No significant difference for the adjusted prevalence of PAD in all segmented categories was observed from that in individuals with microalbuminuria (Figure 3). Significant linear trends in the association between segmented UACR categories and all atherosclerotic conditions were found $\left(P_{\text {trend }}<0.001\right.$ for all).

\section{Diagnostic performance of UACR in predicting abnormal atherosclerosis}

The area under the curve (AUC), which measures the discriminatory ability of the test to classify correctly those with and without the outcome, was highest in individuals with abnormal BaPWV [AUC $=0.675$; 95\% confidence interval (CI), 0.661-0.689] and lowest in those with abnormal CCA IMT (AUC $=0.578 ; 95 \%$ CI, 0.560-0.596). When Youden's indices were calculated for selected cut-off values of $5,10,15,20,25$, and $30 \mathrm{mg} / \mathrm{g}$, the indices were greatest for the cut-off value of $15 \mathrm{mg} / \mathrm{g}$ in all abnormal atherosclerotic conditions. More exactly, the best cut-off values, showing the highest sensitivity and specificity, were 16.4, 15.8, 14.2, 14.7, and $18.4 \mathrm{mg} / \mathrm{g}$ for abnormal CCA IMT, abnormal CCA diameter, abnormal $\mathrm{BaPWV}$, carotid plaques, and PAD, respectively (Table 2). 


\section{Kidney Blood Pressure Research}

Fig. 2. Adjusted mean $(95 \%$ interval) of atherosclerotic parameters [common carotid artery (CCA) intima-media thickness (IMT) and diameter, brachial-ankle pulse wave velocity (BaPWV)] according to urinary albumin-tocreatinine ratio (UACR). Means were adjusted for age, sex, body mass index, smoking status, alcohol consumption, hy-pertension, diabetes, t o tal / high-den sity lipoprotein cholesterol ratio, use of lipidlowering medication, and uric acid.

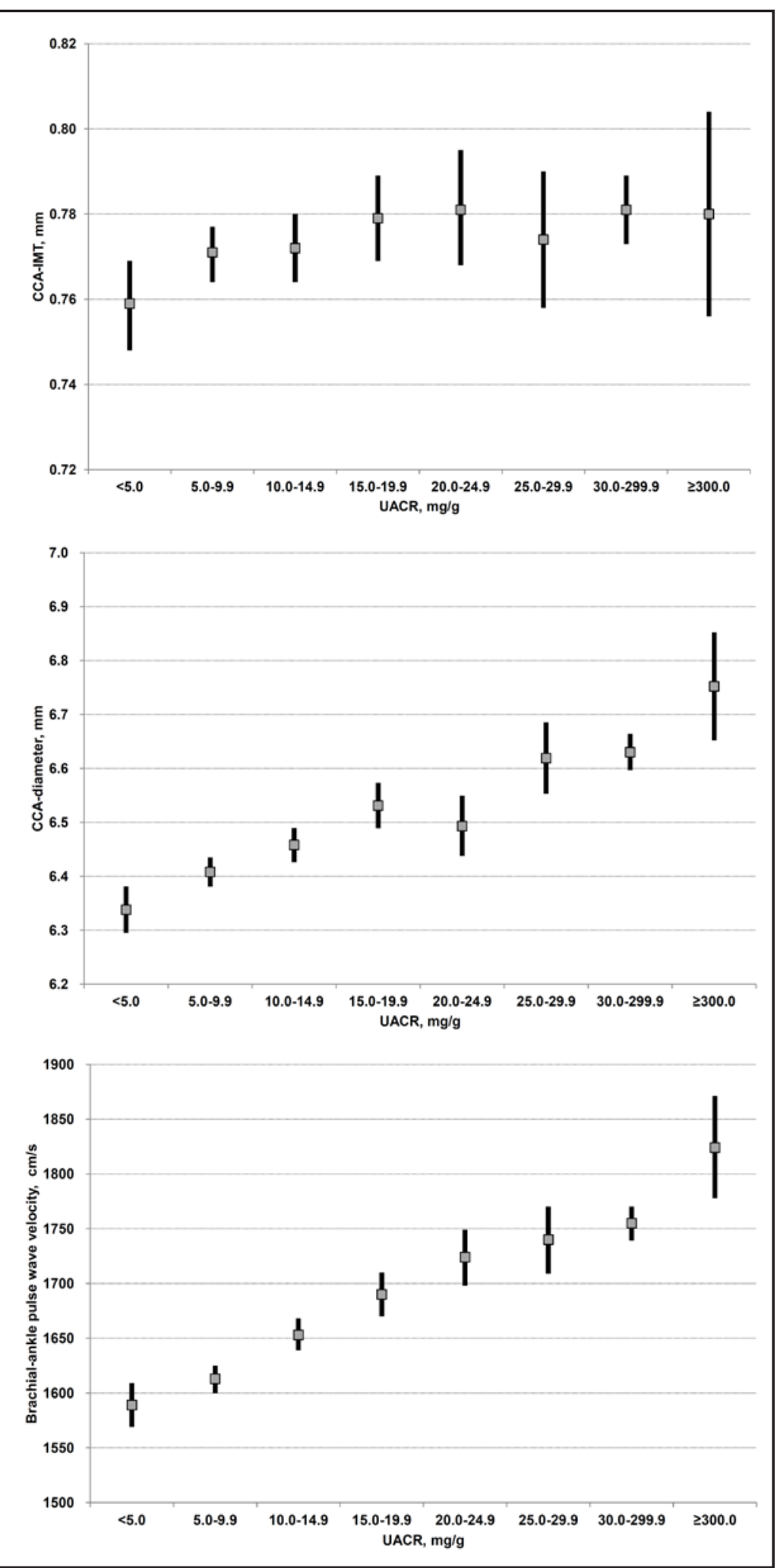

In additional separate analyses of men and women, the optimal cut-off values of the UACR were similar in both sexes, at $15 \mathrm{mg} / \mathrm{g}$. The best cut-off points of the UACR that predicted abnormal atherosclerotic vascular disease were $14-18 \mathrm{mg} / \mathrm{g}$ in men and 15-18 $\mathrm{mg} / \mathrm{g}$ in women (data not shown). 


\section{Kidney Blood Pressure Research}

Fig. 3. Adjusted prevalence $(95 \%$ confidence interval) of carotid plaques and peripheral arterial disease (PAD) according to urinary albumin-tocreatinine ratio (UACR). Prevalence was adjusted for age, sex, body mass index, smoking status, alcohol consumption, hypertension, diabetes, total/ high-density lipoprotein cholesterol ratio, use of lipid-lowering medication, and uric acid.

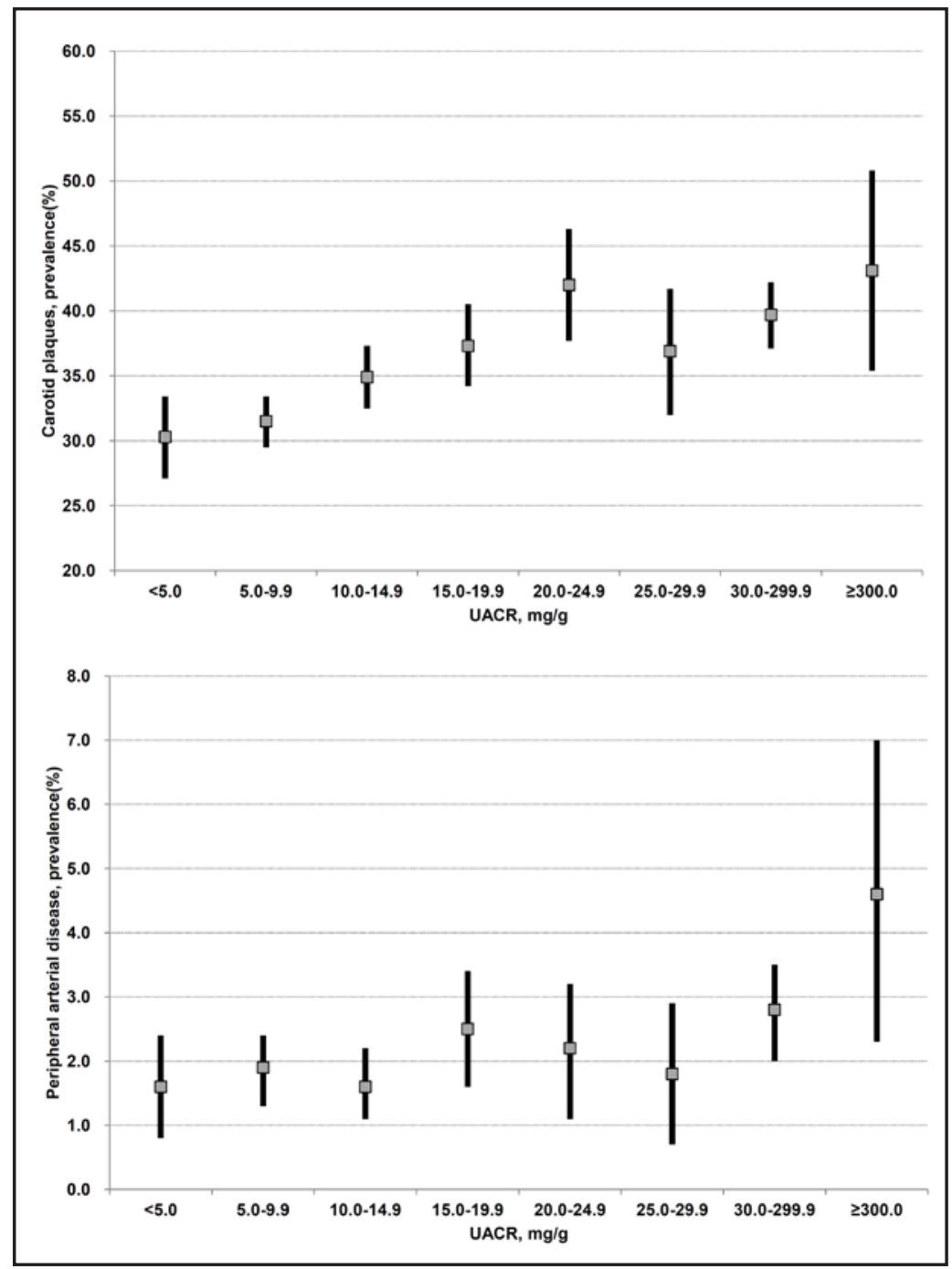

Revised classification of UACR with atherosclerotic parameters

Adding the new cut-off value of $15.0 \mathrm{mg} / \mathrm{g}$, the UACR was reclassified to define low normoalbuminuria, high normoalbuminuria, microalbuminuria, and macroalbuminuria. Compared with low normoalbuminuria, CCA IMT, CCA diameter, and BaPWV were significantly greater in those with high normoalbuminuria in both models 1 and 2 . The risk of carotid plaques was significantly higher in individuals with high normoalbuminuria than in those with low normoalbuminuria in model 1 [odds ratio (OR), 1.39; 95\% CI, 1.24-1.56] and model 2 (OR, 1.30; 95\% CI, 1.16-1.46). The risk of PAD was significantly higher in people with high normoalbuminuria than in those with low normoalbuminuria only in age- and sexadjusted models (OR, 1.42; 95\% CI, 1.03-1.96) (Table 3).

\section{Discussion}

This cross-sectional study of a community-dwelling population demonstrated that the UACR was significantly associated with atherosclerotic vascular diseases. Because atherosclerotic vascular parameters were significantly increased according to UACR, even in the conventional normoalbuminuria range, this study proposed a new UACR cut-off value of $15.0 \mathrm{mg} / \mathrm{g}$ (replacing $30 \mathrm{mg} / \mathrm{g}$ ) to define the lower limit of microalbuminuria. Compared 


\section{Kidney \\ Blood Pressure Research}

with those with low normoalbuminuria (UACR $<15.0 \mathrm{mg} / \mathrm{g}$ ), individuals with high normoalbuminuria (UACR 15.0$30.0 \mathrm{mg} / \mathrm{g}$ ) had significantly greater CCA IMT, CCA diameter, BaPWV, and risk for carotid plaque. The significant association between albuminuria below the current microalbuminuria level and atherosclerotic vascular disease is in agreement with studies that have shown an association between low-grade albuminuria below thresholds conventionally used to define microalbuminuria and cardiovascular disease (CVD) $[3,4,17]$.

Epidemiological studies have found a significant association between microalbuminuriaand carotid atherosclerosis in patients with hypertension or diabetes [7, 8]. Recent studies have found that the UACR is independently associated with carotid IMT and plaques in the general population $[6,9]$. Rodondi et al. showed that microalbuminuria is associated with carotid atherosclerosis measured by IMT [18]. A communitybased study has also shown a significant relationship between the UACR and carotid IMT in the Korean general population [19]. Low-grade albuminuria, below the current cut-off value for microalbuminuria, is positively associated with CCA IMT in patients with type 2 diabetes [20]. In the general population, a highly significant doseresponse relationship between the UACR and CCA IMT was observed, even at levels far below those conventionally defined as microalbuminuria [9]. However, in the MultiEthnic Study of Atherosclerosis (MESA), no significant difference was observed in CCA IMT between high normal UAE (9.0-16.9 $\mathrm{mg} / \mathrm{g}$ in men, $13.0-24.9 \mathrm{mg} / \mathrm{g}$ in women) and normal UAE $(<9.0 \mathrm{mg} / \mathrm{g}$ in men, $<13.0 \mathrm{mg} / \mathrm{g}$ in women) [10]. A significant dose-response association between UACRs far below those conventionally defined as microalbuminuria and carotid plaque was observed in the general population [9]. In a populationbased prospective study, a linear relationship was found between low UACR and the development of carotid plaques [6]. We confirmed the dose-response relationship of the UACR with carotid atherosclerosis in this study.

To compensate for arterial wall thickening and carotid plaque formation, the

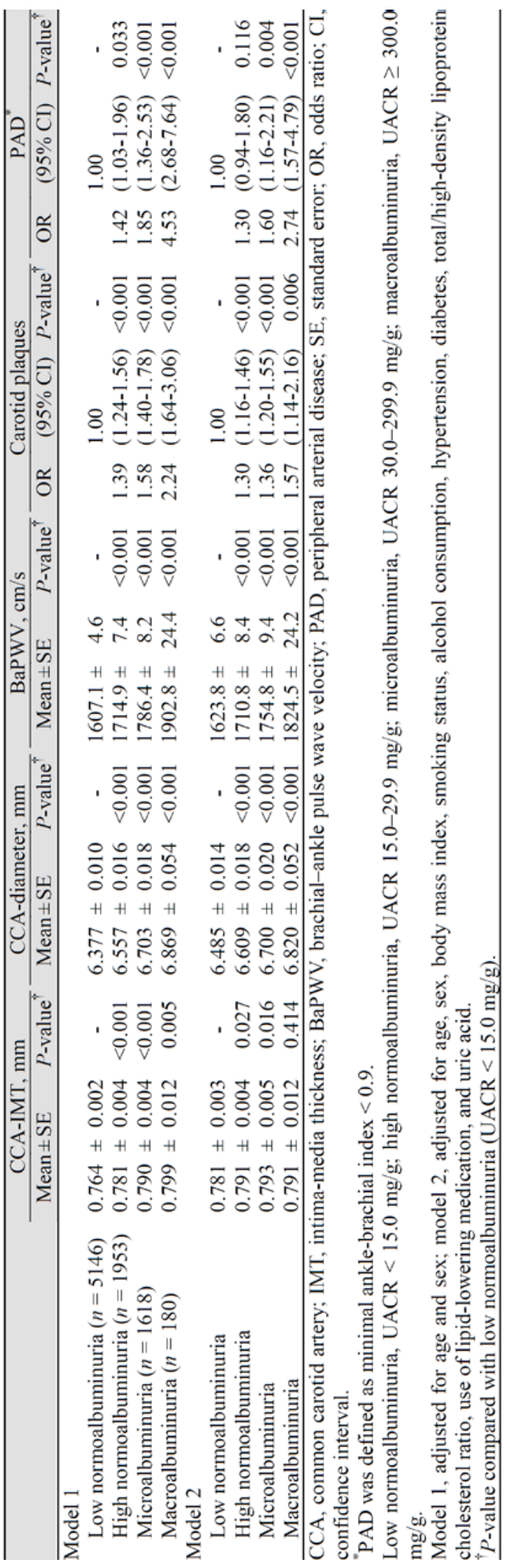

Table 3: Association of the revised classification based on the urinary albumin-to-creatinine ratio (UACR) with atherosclerotic conditions 


\section{Kidney Blood Pressure Research}

carotid artery may enlarge and stabilize the shear stress [21]. Carotid arterial distension is considered to be a surrogate marker of CVD. Our results showing a positive dose-response relationship between increasing UACR and CCA diameter correspond with those of earlier studies, which have reported that the UACR is significantly associated with CCA luminal diameter [22].

PWV has been regarded as an index of arterial stiffness, which is an independent risk factor for cardiovascular events and related death [23], and is known as a prognostic predictive factor [24]. BaPWV is a simple and noninvasive method for determining arterial stiffness, and has been developed for screening vascular damage in large populations. Previous studies have examined the significant association between microalbuminuria and arterial stiffness measured by BaPWV in patients with hypertension or diabetes $[11,12]$. Recent findings in the general population are in agreement with those of previous studies $[13,14]$. The results of the present study are in close agreement with those of many other studies.

The ABI is considered to be a surrogate marker of generalized atherosclerosis because low levels predict CVD and death $[25,26]$. The present study found that albuminuria was independently associated with PAD in the general population. Although previous studies have reported a significant association between microalbuminuria and PAD, defined as low ABI, this association was observed only in a small number of patients with diabetes [27]. One study has reported that albuminuria was independently associated with PAD in diabetic subjects (OR, 1.65; 95\% CI, 1.00-2.74) but not in nondiabetic patients (OR, 1.13; 95\% CI, 0.76-1.65) [28]. In contrast, another study has shown a significant association between albuminuria and PAD in nondiabetic patients (OR, 1.87; 95\% CI, 1.38-2.52) but not in diabetic patients (OR, 1.08; 95\% CI, 0.68-1.73) [29].

To investigate the optimal cut-off value for predicting subclinical atherosclerosis, we used Youden's index with an ROC curve. Youden's indices were greatest for a UACR cut-off value of $\sim 15 \mathrm{mg} / \mathrm{g}$, below the current threshold for microalbuminuria. For a UACR of $30.0 \mathrm{mg} / \mathrm{g}$, the existing lower limit of microalbuminuria, sensitivity was very low. The Italian community study proposed a new UACR cut-off value of $11.5 \mathrm{mg} / \mathrm{g}$ for predicting left ventricular hypertrophy using the ROC curve [5]. Maximizing the sum of sensitivity and specificity is not always the best method to find the optimal lower limit, therefore, cautious selection of a cutoff value with regard to confirmation of costs of false-positive and false-negative results is necessary. However, when considering the significance of microalbuminuria in screening for CVD, the conventional lower limit of microalbuminuria needs to be reduced [5]. The MESA study of an American population suggested a UACR of $9.0 \mathrm{mg} / \mathrm{g}$ for men and 13.0 for women, which are lower than the conventional thresholds, as new cut-off values that were strongly associated with subclinical CVD [10].

Epidemiological studies have shown that low-grade albuminuria is associated with an increased risk for CVD and death. In nonhypertensive and nondiabetic individuals, UACRs at or above the sex-specific median ( $\geq 3.9 \mathrm{mg} / \mathrm{g}$ for men and $\geq 7.5 \mathrm{mg} / \mathrm{g}$ for women) resulted in a threefold increased risk for CVD compared with patients with UACRs below the median [3]. In the general population, a prospective study found that UAE $>4.8 \mu \mathrm{g} / \mathrm{min}$ was associated with a significant, almost twofold increased risk of coronary heart disease and death [4]. In hypertensive patients, the relative risks of coronary heart disease and death associated with UAE $>5 \mu \mathrm{g} / \mathrm{min}$ were 2.0 and 1.9 , respectively [17]. Moreover, recent investigations have demonstrated that reduced albuminuria is associated with a reduced incidence of cardiovascular events [30].

The present study had several limitations. First, the study was cross-sectional, and thus cannot be used to infer causality. Therefore, the present findings need to be confirmed in a further longitudinal cohort study with a larger population. Second, urinary albumin was measured on only a single urine specimen. Although 24-h urine collection is the gold standard for the diagnosis of microalbuminuria, it is not a good choice for the general population. An accurate diagnosis of microalbuminuria requires an elevated UACR in two of three samples of first morning urine. 


\section{Kidney \\ Blood Pressure Research}

Kidney Blood Press Res 2012;36:290-300

DOI: $10.1159 / 000343418$

Published online: December 12, 2012

(C) 2012 S. Karger AG, Basel

www.karger.com/kbr

Lee/Kweon/Choi et al.: Albuminuria and Atherosclerosis

\section{Conclusion}

Subclinical carotid atherosclerosis and arterial stiffness have already developed in individuals with low-grade albuminuria (UACR $15.0-29.9 \mathrm{mg} / \mathrm{g}$ ), which is currently considered as normal, compared with UACR $<15.0 \mathrm{mg} / \mathrm{g}$. Further longitudinal studies are needed to examine the relationship between microalbuminuria and the development of subclinical atherosclerosis and prognosis of coronary artery disease.

\section{Conflict of Interests}

All authors declared no competing interests.

\section{Acknowledgments}

This study was supported by Wonkwang University in 2011.

\section{References}

1 Keane WF, Eknoyan G: Proteinuria, albuminuria, risk, assessment, detection, elimination (PARADE): a position paper of the National Kidney Foundation. Am J Kidney Dis 1999;33:1004-1010.

-2 Hillege HL, Fidler V, Diercks GF, van Gilst WH, de Zeeuw D, van Veldhuisen DJ, Gans RO, Janssen WM, Grobbee DE, de Jong PE, Prevention of Renal and Vascular End Stage Disease (PREVEND) Study Group: Urinary albumin excretion predicts cardiovascular and noncardiovascular mortality in general population. Circulation 2002;106:1777-1782.

-3 Arnlöv J, Evans JC, Meigs JB, Wang TJ, Fox CS, Levy D, Benjamin EJ, D‘Agostino RB, Vasan RS: Low-grade albuminuria and incidence of cardiovascular disease events in nonhypertensive and nondiabetic individuals: the Framingham Heart Study. Circulation 2005;112:969-975.

-4 Klausen K, Borch-Johnsen K, Feldt-Rasmussen B, Jensen G, Clausen P, Scharling H, Appleyard M, Jensen JS: Very low levels of microalbuminuria are associated with increased risk of coronary heart disease and death independently of renal function, hypertension, and diabetes. Circulation 2004;110:32-35.

5 Ratto E, Leoncini G, Viazzi F, Vaccaro V, Parodi A, Falqui V, Bezante GP, Tomolillo C, Deferrari G, Pontremoli R: Microalbuminuria and cardiovascular risk assessment in primary hypertension: should threshold levels be revised? Am J Hypertens 2006;19:728-734.

6 Jørgensen L, Jenssen T, Johnsen SH, Mathiesen EB, Heuch I, Joakimsen O, Fosse E, Jacobsen BK: Albuminuria as risk factor for initiation and progression of carotid atherosclerosis in non-diabetic persons: the Troms $\varnothing$ Study. Eur Heart J 2007;28:363-369.

7 Yokoyama H, Aoki T, Imahori M, Kuramitsu M: Subclinical atherosclerosis is increased in type 2 diabetic patients with microalbuminuria evaluated by intima-media thickness and pulse wave velocity. Kidney Int 2004;66:448-454.

8 Bigazzi R, Bianchi S, Nenci R, Baldari D, Baldari G, Campese VM: Increased thickness of the carotid artery in patients with essential hypertension and microalbuminuria. J Hum Hypertens 1995;9:827-833.

-9 Furtner M, Kiechl S, Mair A, Seppi K, Weger S, Oberhollenzer F, Poewe W, Willeit J: Urinary albumin excretion is independently associated with carotid and femoral artery atherosclerosis in the general population. Eur Heart J 2005;26:279-287.

10 Kramer H, Jacobs DR Jr, Bild D, Post W, Saad MF, Detrano R, Tracy R, Cooper R, Liu K: Urine albumin excretion and subclinical cardiovascular disease. The Multi-Ethnic Study of Atherosclerosis. Hypertension 2005;46:38-43.

11 Liu CS, Pi-Sunyer FX, Li CI, Davidson LE, Li TC, Chen W, Lin CC, Huang CY, Lin WY: Albuminuria is strongly associated with arterial stiffness, especially in diabetic or hypertensive subjects--a population-based study (Taichung Community Health Study, TCHS). Atherosclerosis 2010;211:315-321. 


\section{Kidney \\ Blood Pressure Research}

12 Munakata M, Nunokawa T, Yoshinaga K, Toyota T, J-TOPP Study Group: Brachial-ankle pulse wave velocity is an independent risk factor for microalbuminuria in patients with essential hypertension-a Japanese trial on the prognostic implication of pulse wave velocity (J-TOPP). Hypertens Res 2006;29:515-521.

13 Kim BJ, Lee HA, Kim NH, Kim MW, Kim BS, Kang JH: The association of albuminuria, arterial stiffness, and blood pressure status in nondiabetic, nonhypertensive individuals. J Hypertens 2011;29:2091-2098.

-14 Ishikawa T, Hashimoto J, Morito RH, Hanazawa T, Aikawa T, Hara A, Shintani Y, Metoki H, Inoue R, Asayama K, Kikuya M, Ohkubo T, Totsune K, Hoshi H, Satoh H, Imai Y: Association of microalbuminuria with brachialankle pulse wave velocity: the Ohasama study. Am J Hypertens 2008;21:413-418.

-15 Choi SW, Kim HY, Lee YH, Ryu SY, Kweon SS, Rhee JA, Choi JS, Shin MH: eGFR is associated with subclinical atherosclerosis independent of albuminuria: the Dong-gu Study. Atherosclerosis 2010;212:661-667.

-16 Selvin E, Erlinger TP: Prevalence of and risk factors for peripheral arterial disease in the United States: results from the National Health and Nutrition Examination Survey 1999-2000. Circulation 2004;110:738743.

17 Klausen KP, Scharling H, Jensen G, Jensen JS: New definition of microalbuminuria in hypertensive subjects: association with incident coronary heart disease and death. Hypertension 2005;46:33-37.

18 Rodondi N, Yerly P, Gabriel A, Riesen WF, Burnier M, Paccaud F, Bovet P: Microalbuminuria, but not cystatin C, is associated with carotid atherosclerosis in middle-aged adults. Nephrol Dial Transplant 2007;22:11071114.

19 Sung JK, Kim JY, Youn YJ, Lee JW, Ahn SG, Yoo BS, Lee SH, Yoon J, Choe KH, Yoon JH, Park JK, Koh SB: Urine Albumin Creatinine Ratio is Associated with Carotid Atherosclerosis in a Community Based Cohort: Atherosclerosis Risk of Rural Area in Korean General Population Study. J Cardiovasc Ultrasound 2010;18:134-138.

20 Huang Y, Chen Y, Xu M, Gu W, Bi Y, Li X, Ning G: Low-grade albuminuria is associated with carotid intimamedia thickness in Chinese type 2 diabetic patients. J Clin Endocrinol Metab 2010;95:5122-5128.

-21 Labropoulos N, Zarge J, Mansour MA, Kang SS, Baker WH: Compensatory arterial enlargement is a common pathobiologic response in early atherosclerosis. Am J Surg 1998;176:140-143.

-22 Hermans MM, Henry RM, Dekker JM, Nijpels G, Heine RJ, Stehouwer CD: Albuminuria, but not estimated glomerular filtration rate, is associated with maladaptive arterial remodeling: the Hoorn Study. J Hypertens 2008;26:791-797.

23 Lehmann ED: Clinical value of aortic pulse-wave velocity measurement. Lancet 1999;354:528-529.

24 Laurent S, Boutouyrie P: Arterial stiffness: a new surrogate end point for cardiovascular disease? J Nephrol 2007;20:S45-S50.

25 Abbott RD, Petrovitch H, Rodriguez BL, Yano K, Schatz IJ, Popper JS, Masaki KH, Ross GW, Curb JD: Ankle/ brachial blood pressure in men $>70$ years of age and the risk of coronary heart disease. Am J Cardiol 2000;86:280-284.

-26 Vogt MT, Cauley JA, Newman AB, Kuller LH, Hulley SB: Decreased ankle/arm blood pressure index and mortality in elderly women. JAMA 1993;270:465-469.

27 Kallio M, Forsblom C, Groop PH, Groop L, Lepäntalo M: Development of new peripheral arterial occlusive disease in patients with type 2 diabetes during a mean follow-up of 11 years. Diabetes Care 2003;26:12411245.

28 Wattanakit K, Folsom AR, Criqui MH, Kramer HJ, Cushman M, Shea S, Hirsch AT: Albuminuria and peripheral arterial disease: results from the multi-ethnic study of atherosclerosis (MESA). Atherosclerosis 2008;201:212-216.

-29 Wu CK, Yang CY, Tsai CT, Chiu FC, Huang YT, Lee JK, Cheng CL, Lin LY, Lin JW, Hwang JJ, Chiang FT: Association of low glomerular filtration rate and albuminuria with peripheral arterial disease: the National Health and Nutrition Examination Survey, 1999-2004. Atherosclerosis 2010;209:230-234.

30 Brouwers FP, Asselbergs FW, Hillege HL, de Boer RA, Gansevoort RT, van Veldhuisen DJ, van Gilst WH: Longterm effects of fosinopril and pravastatin on cardiovascular events in subjects with microalbuminuria: Ten years of follow-up of Prevention of Renal and Vascular End-stage Disease Intervention Trial (PREVEND IT).

-Am Heart J 2011;161:1171-1178. 